\title{
State Space Estimation for an Electromechanical Actuator Valve using Extended Kalman Filter
}

\author{
Zs. Horváth \\ Production Planning V-Engines, \\ AUDI Hungaria Motor Kft., 9027 Győr, Kardán utca 1. \\ zsolt2.horvath@audi.hu
}

\begin{abstract}
This paper discusses a state space estimation for an electromechanical actuator valve using extended Kalman filter (EKF). A proposed actuator model includes a Tustin's friction model with strong nonlinearities, hence it represents an accurate model for describing the friction phenomenon in an electromechanical actuator valve. At first the state equations of the model were converted into a time-discrete. Then the EKF is used for estimation of three states, namely the motor current, valve angular velocity - and position. Both the actuator model and the EKF algorithm were implemented in MATLAB ${ }^{\circledR}$. The measurements for input data in the EKF are performed in National Instruments CompactRIO system. Outline of our examination should be to take a conclusion, how much EKF is capable of accurate estimation of the desired states. Furthermore we will use this method for further developing the fault detection in the electromechanical actuator valve.
\end{abstract}

Keywords: extended Kalman filter, electromechanical actuator valve, state space estimation, Tustin's friction model

\section{Introduction}

Processing integrated electromechanical systems such as actuators and sensors are fundamental part of automotive vehicles. With increasing complexity of them, the methodology of state space estimation becomes an essential part of the field of fault diagnosis and condition monitoring. Generally the electromechanical actuators product cost such as the electromechanical throttle valve must be low. On the other hand their structure is complex and they have a relatively large number of components. The measurement of desired state signals such as current, pressure, velocity, position are often physically not possible or application of additional sensors in them is too expensive.

A typical case in most dynamical systems with complex structure is that the state vector can not be measured. In these situations a suitable approximation to the state 
vector is needed. The extended Kalman filter is a very frequently used tool for performing state estimation on nonlinear dynamical systems.

This paper discusses a state space estimation for an electromechanical actuator valve using extended Kalman filter (EKF). A Tustin's friction model can be found in our proposed actuator valve model which includes nonlinearities too, that is why it represents an accurate model in an electromechanical actuator valve for describing the friction phenomenon. It is necessary because the EKF requires an accurate model of the plant. In this work I have used the EKF for the estimation of the motor current, valve angular velocity - and position.

For modelling and control of electromechanical actuators nowadays are used many different observer concepts. A nonlinear Variable Structure System (VSS) observer for throttle systems is presented in [1]. Paper [2] uses a sliding-mode observer for a robust position control of these actuators. An additional study is [3], it describes applying Luenberger observer for sensor monitoring in active front steering systems. Another study [4] is about using the Kalman filter for the observation of the DC-motor. The most common approaches are using extended Kalman filter (EKF) or unscented Kalman filter (UKF) [5]. These two methods are preferable solutions in our case now too, when the process nonlinearities are strong and noise is associated with the real system. Research [6] used the EKF for parameter estimation of a hydraulic proportional valve. Another research [7] extended it to fault detection.

The throttle valve is a type of an electromechanical actuator, it is advanced in several applications of combustion engine control (intake manifold, exthaust gas recirculation, variable turbine geometry, ect.).

The presented paper consists of : the electromechanical throttle valve model is introduced in Section I, Section II is about the design of the extended Kalman filter, Section III shows the experimental results and finally Section IV presents the main obtained results.

\section{Basic Model of an Electromechanical Throttle Valve}

The electromechanical throttle valve (Fig. 1.) regulates air flow in the engine of the car. It consists of following parts: DC-motor, gearbox, return spring, throttle plate and sensor for valve position. A bipolar chopper supplies the DC-motor, a gearbox to the throttle plate transmitts the motor shaft rotation. The position of the throttle plate is given to the ECU (Engine Control Unit). A reset spring places the de-energized throttle plate in its default position (,limp-home”). The valve is slightly opened here, and the engine can operate at minimum power.

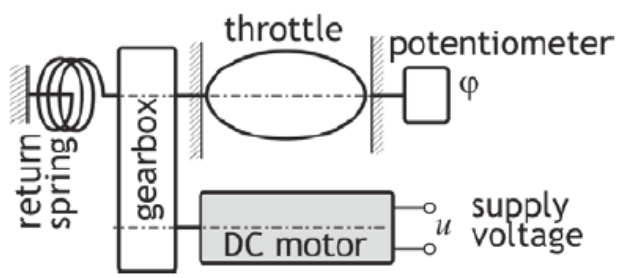

Figure 1. The scheme of the electromechanical actuator [8] 


\subsection{Model Equations of the Electromechanical Throttle Valve}

The relation between input voltage $u_{a}$ and current $i$ in the armature circuit can be described as

$$
u_{a}=R i+L \frac{d i}{d t}+K_{e} n \omega .
$$

where $L$ is the inductance, $R$ is the resistance in the armature circuit, $K_{e}$ is the inductive voltage constant, $n$ is the gear ratio and $\omega$ is the angular velocity of the throttle plate.

The throttle valves are usually equipped with two springs. One spring is acting on the valve when the angular positon is greather than the „limp-home” position, whereas the other is acting when the angular position is smaller than the „limp-home” position. In our approach we have considered that the two springs may have some spring stiffness. The signal of the spring pretension torque will be changed depending on the signal of the angular position of the throttle. Further in our model we assume the „limp-home” postion at angular position by zero. The realisation of the spring torque is depicted on the Fig. 2.

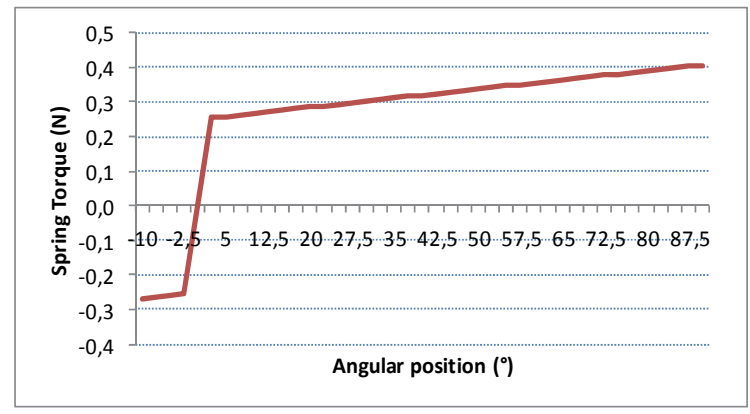

Figure 2. Nonlinear spring torque

The motion Equations of the throttle plate (related to the plate axel) can be desribed as

$$
J \frac{d \omega}{d t}=K_{t} i-M_{o} \operatorname{sign}(\varphi)-c \varphi-M_{f} .
$$

where $\varphi$ is the angular position of the throttle plate, $J$ is the rotary inertia at the throttle shaft, which is composed of inertia of the DC-motor. $M_{o}$ denotes a torque due to the prestressed springs. Finally $K_{t}$ denotes the motor torque constant.

When the Stribeck effect is taken in consideration, the friction model is called Tustin's model. The Tustin's model is defined as

$$
M_{f}=\left[M_{c}+\left(\mathrm{M}_{s}-M_{c}\right) e^{-|\mathrm{v}| / \mathrm{v}_{s}}\right] \operatorname{sign}(\dot{x})+k_{v} \dot{\varphi}
$$


where

$M_{f}$ denotes the static friction torque, $M_{c}$ is a torque of the coulomb friction of the system, $M_{s}$ is the stiction force, $v$ represents the velocity, $v_{s}$ is the Stribeck velocity, $c$ denotes a linear spring constant. $K_{v}$ is a positive constant for viscousus friction.

Table 1. EMA parameters

\begin{tabular}{|c|c|c|}
\hline Parameter & Values & Units \\
\hline$R$ & 4.3 & $\mathrm{Ohm}$ \\
\hline$L$ & 0.002 & $\mathrm{H}$ \\
\hline$K_{e}$ & 0.017 & $\mathrm{Vs} / \mathrm{rad}$ \\
\hline$K_{t}$ & 0.017 & $\mathrm{Nm} / \mathrm{A}$ \\
\hline$J$ & 0.0016 & $\mathrm{kgm}$ \\
\hline$n$ & 40 & - \\
\hline$M_{c}$ & 0.2 & $\mathrm{Nm}$ \\
\hline$M_{0}$ & 0.25 & $\mathrm{Nm}$ \\
\hline$c$ & 0.059 & $\mathrm{Nm} / \mathrm{rad}$ \\
\hline$K_{v}$ & 0.01 & $\mathrm{Nms} / \mathrm{rad}$ \\
\hline$M_{S}$ & 0.3 & $\mathrm{Nm}$ \\
\hline$v_{s}$ & 1 & $\mathrm{rad} / \mathrm{sec}$ \\
\hline
\end{tabular}

Table 1 shows the values of the model parameters, some of them $\left(R, L, K e, K t, J, M_{0}\right.$, $c$ ) are provided by the manufacturer (Continental AG). The remaining parameters are identified with the well known identifications procedures, with the static load test and viscous frictional coeffecient test, see e.g. [9].

\subsection{Discrete State Space Model of the Electromechanical Throttle Valve}

A discrete state space model of the system is required for applying the extended Kalman filter. Hence at first the state equations of the model must be converted into a time-discrete.

With having system and measurement noise matrices as $w(t)$ and $v(t)$ respectively, the continuous state space model for a linear system can be written as :

$$
\begin{gathered}
\dot{X}(t)=A X(t)+B U(t)+w(\mathrm{t}), \\
Z(t)=C X(t)+v(t) .
\end{gathered}
$$

where $X(t)$ is the state vector, (n x 1) matrix, $A$ is the system, (n x n) matrix, $B$ is the input matrix, (n x p), $C$ is the output matrix, $(\mathrm{mx} \mathrm{n}), U(t)$ is the input vector, (p x 1), $Z(t)$ is the output vector, $(\mathrm{m} \times 1), w(t)$ is the system noise, $(\mathrm{p} \times 1)$, and $v(t)$ represents the measurement noise, ( $\mathrm{m} \times 1)$.

To get a discrete state space model, I have used the "forward difference" approximation to calculate the derivative, which given by 


$$
\dot{X}(t) \approx \frac{X(k+1)-X(k)}{T_{s}} .
$$

where $T_{s}$ is the sampling time, $k$ is a step, $(k+1)$ is a next step of the iteration.

Using the Equation (6) and applying it to the Equations (4), (5), the discrete state space model is [7]:

$$
\begin{gathered}
X(k+1)=\Phi X(k)+G U(k)+W(\mathrm{k}), \\
Z(k)=H X(k)+V(k), \\
\text { with } \Phi(k)=T_{s} A+1, \\
G(k)=T_{s} B, \\
W(k)=T_{s} w(k), \\
V(k)=v(k) .
\end{gathered}
$$

where $\Phi$ is the discrete system matrix, $G$ represents the discrete input matrix, $H$ stands for the discrete output matrix, $W(k)$ represents the process noise and $V(k)$ is the measurement noise. It is assumed, that process noise and measurement noise (elements of $W(k)$ and $V(k)$ ) are respectively uncorrelated and white .

Thus,

$$
W \approx N(0, Q(k)), \text { and } V \approx N(0, R(k))
$$

where $Q(k)$ and $R(k)$ are known covariance matrices of $W(k)$ and $V(k)$ respectively.

Finally we define the noise covariance matrices as

$$
\begin{array}{rlrl}
E\left[W_{i}(k) W_{j}(k)^{T}\right] & =Q(k), & i=j, \\
& =0, & i \neq j, \\
E\left[V_{i}(k) V_{j}(k)^{T}\right] & =R(k), \quad i=j, \\
& =0, & i \neq j .
\end{array}
$$

Using the forward difference approximation Equation (6) and applying it to the continuous state space model Equations (1) to (3), the discrete state space model of the electromechanical throttle valve is given by 


$$
\begin{aligned}
& x_{1}(k+1)=x_{1}(k)-\frac{R}{L} x_{1}(k) T_{s}-n \frac{K_{e}}{L} x_{2}(k) T_{s}+\frac{1}{L} U(k) T_{s}+w_{1}(k) \\
& x_{2}(k+1)=\frac{n K_{t}}{J} x_{1}(k) T_{s}+x_{2}(k)-\frac{K_{v}}{J} x_{2}(k) T_{s}-\frac{c}{J} x_{3}(k) T_{s} \\
& -\frac{M_{0}}{J} \operatorname{sign}\left(x_{3}(k)\right) T_{s}-\frac{1}{J}\left(M_{c}+\left(M_{s}-M_{c}\right) e^{\left.-\mid \frac{\omega}{\omega_{s} \mid}\right)} \operatorname{sign}\left(x_{2}(k)\right) T_{s}\right. \\
& +M_{\text {Stop }}(\varphi, \omega)+w_{2}(k) \\
& x_{3}(k+1)=x_{2}(k) T_{s}+x_{3}(k)+w_{3}(k) .
\end{aligned}
$$

where $x_{1}(k)$ is the state variable for motor current, $x_{2}(k)$ is a state variable for angular speed of the throttle, $x_{3}(k)$ is the state variable for trottle angle, $w_{1}, w_{2}, w_{3}$, are the system noise elements, $T_{s}$ is the sampling time.

\section{Applying Extended Kalman Filter to State Estimation of the Electromechanical Throttle Valve}

\subsection{Extended Kalman Filter}

To perform state estimation on nonlinear dynamical systems, the extended Kalman filter is a very commonly used tool. The extended Kalman filter is used for parameter and state estimation, it is a modified form of the Kalman filter. The Kalman filter is an optimal state estimation process applied to dynamic systems with random perturbations. If the process model is nonlinear, the Kalman filter is applied by continually updating the linearization around the most recent reference $X(k)$ for the system function and $X^{-}(k)$ for the measurement function as shown on the Figure 3.

A Taylor approximation of the system function is made at the refined state estimate $\hat{X}(k)$ and an approximation of the measurement function is made at the unrefined state estimate $\hat{X}^{-}(k)$.

The nonlinear model can be represented with:

$$
\begin{gathered}
X(k+1)=f(X(k), U(k))+W(k), \\
Z(k)=h(X(k))+V(k) .
\end{gathered}
$$

where $f(X(k), U(k))$ and $h(X(k))$ are the nonlinear system and measurement functions respectively.

The linearization is made using the most recent obtained state estimate, which is using the unrefined estimate for the system function and the refined estimate for the measurement function. 
Therefore, doing a linear Taylor series approximation of $f(X(k), U(k))$ at an operating point $\hat{X}(k)$ and of $h(X(k))$ at the operating point $\hat{X}^{-}(k)$, and ignoring the higher-level term yields, the linearised form is:

$$
\begin{gathered}
X(k+1)=\Phi(k) X(k)+U_{1}(k)+W(k), \\
Y(k)=H(k) X(k)+V(k),
\end{gathered}
$$

where:

$$
\begin{gathered}
\Phi(k)=\left.\frac{\partial f(X(k), U(k))}{\partial X(k)}\right|_{\hat{X}(k)}, \\
H(k)=\left.\frac{\partial h(X(k))}{\partial X(k)}\right|_{\hat{X}^{-}(k)}, \\
U_{1}(k)=f(\hat{X}(k), U(k))-\Phi(k) \hat{X}(k), \\
Y(k)=Z(k)-h\left(\hat{X}^{-}(k)\right)+H(k) \hat{X}^{-}(k) .
\end{gathered}
$$

At step $\mathrm{k}$ a refined estimate of $\hat{X}(k)$ is now used to predict what $\hat{X}^{-}(k)$ is at step $k+1$, based on the plants modeled dynamics.

The five Kalman filtering equations can be summarized as follows:

Estimation equations:

$$
\begin{gathered}
K(k)=P^{-}(k) H^{T}\left[H P^{-}(k) H^{T}+R(k)\right]^{-1}, \\
\hat{X}(k)=\hat{X}^{-}(k)+\mathrm{K}(\mathrm{k})\left[Z(k)-H \hat{X}^{-}(k)\right], \\
P(k)=[I-K(k) H] P^{-}(k) .
\end{gathered}
$$

Prediction equations:

$$
\begin{aligned}
& \hat{X}^{-}(k+1)=\Phi \hat{X}(k)+G U(k), \\
& P^{-}(k+1)=\Phi \mathrm{P}(\mathrm{k}) \Phi^{T}+Q(k) .
\end{aligned}
$$

The EKF is a recursive algorithm with a set of prediction (time update) and correction (measurement update) is shown in the Figure 3. 


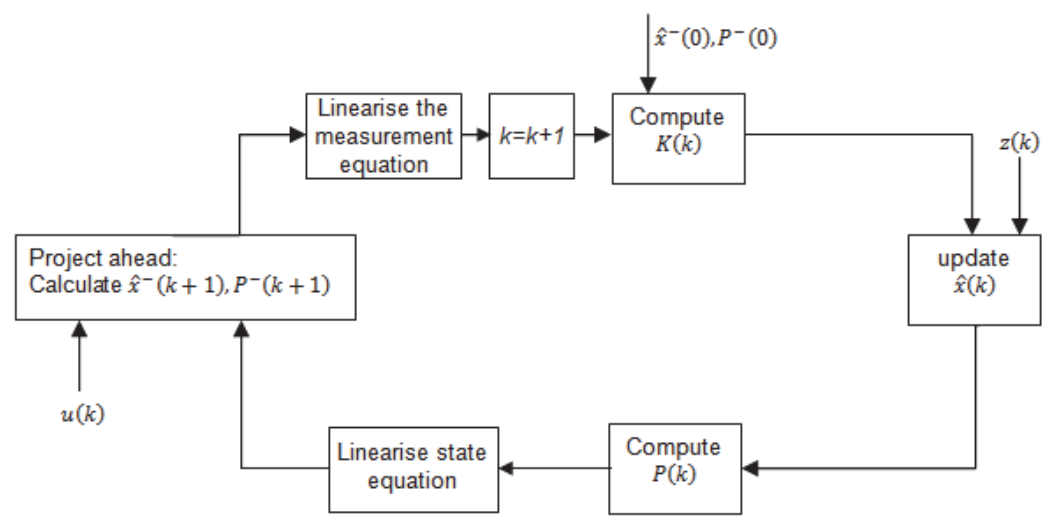

Figure 3. Flowchart of the recursive extended Kalman filter [6]

\subsection{Observability Test}

In state estimation of dynamic systems, observability is one of the most important requirements. In the case when the system is linear, it can be characterized with the rank of the observability matrices, a methology for this can be found in [10]. If the system is nonlinear observability is more complicated. The nonlinear observability is highly dependent on the Lie derivative, which is a derivative of a scalar function along a vector field. We can define the Lie derivative of scalar function $h$ with respect to a vector field $f$, denoted $L_{f}(h)$.

It is given by

$$
L_{f}(h)=\frac{\partial f}{\partial x} f(x)
$$

The examination of the observability is not discussed in this paper, a method for it is presented in [11]. The theory of the observability of nonlinear systems is discussed in [12].

\subsection{State Estimation for the Electromechanical Throttle Valve by the EKF}

As above mentioned, the extended Kalman filter is used to estimate three states of the electromechanical throttle valve, namely motor current, valve angular velocity and valve angular position. In order to apply the EKF, firstly the Equations (15), (16) must be calculated. The discrete output matrix is:

$$
H=\left[\begin{array}{lll}
1 & 0 & 0 \\
0 & 1 & 0 \\
0 & 0 & 1
\end{array}\right],
$$


The output Equation is:

$$
\begin{gathered}
Z(k)=H X(k)+V(k), \\
Z(k)=\left[\begin{array}{ccc}
1 & 0 & 0 \\
0 & 1 & 0 \\
0 & 0 & 1
\end{array}\right]\left[\begin{array}{l}
x_{1}(k) \\
x_{2}(k) \\
x_{2}(k)
\end{array}\right]+\left[\begin{array}{c}
v_{1}(k) \\
v_{2}(k) \\
v_{3}(k)
\end{array}\right] .
\end{gathered}
$$

where $V_{1}(k), V_{2}(k), V_{3}(k)$ are the measurement noise elements.

Just as next step should be the Jacobi matrix calculated, with using Eq. 12. On them the function $\operatorname{sign}(x)$ is not able to take the derivative, because it is not continued at zero.

Therefore we use the approximation $\quad \operatorname{sign}(\mathrm{x}) \approx \frac{2}{\pi} \operatorname{arctg}\left(10^{3} x\right)$,

The linearized (Jacobi) system matrix is:

$$
\Phi(k)=\frac{\partial f(X(k))}{\partial X(k)}=\left[\begin{array}{lll}
\Phi_{11}(k) & \Phi_{12}(k) & \Phi_{13}(k) \\
\Phi_{21}(k) & \Phi_{22}(k) & \Phi_{23}(k) \\
\Phi_{31}(k) & \Phi_{32}(k) & \Phi_{33}(k)
\end{array}\right]
$$

where the elements of the matrix are

$$
\begin{aligned}
& \Phi_{11}=1-\frac{R}{L} T_{s}, \Phi_{12}=n \frac{K_{e}}{L} T_{s}, \Phi_{13}=0, \\
& \Phi_{21}=n \frac{K_{t}}{J} T_{s}, \\
& \Phi_{22}=1-\frac{K_{v}}{J} T_{s}-10^{3} T_{s} \frac{M_{c}+\left(M_{s}-M_{c}\right)}{J \pi\left(1+10^{6} \operatorname{Xest}(2)^{2}\right)} e^{-\left|\frac{\operatorname{Xest}(2)}{\omega_{s}}\right|}, \\
& \Phi_{23}=-\frac{c}{J} T_{s}-\frac{10^{3} T_{s} M_{0}}{J \pi\left(1+10^{6} \operatorname{Xest}(3)^{2}\right)} T_{s}, \\
& \Phi_{31}=0, \Phi_{32}=T_{s}, \Phi_{33}=1 .
\end{aligned}
$$

where Xest denotes a vector, that includes the estimated state space variables.

It is assumed that the initial values of the states has a known mean value and variance. Hence the initial state vector and the initializing matrices were set as follows: 


$$
\begin{gathered}
x_{0}(k)=\left[\begin{array}{lll}
0 & 0 & 0
\end{array}\right]^{T} \\
P(0)=\left[\begin{array}{ccc}
10^{2} & 0 & 0 \\
0 & 10^{2} & 0 \\
0 & 0 & 10^{2}
\end{array}\right] .
\end{gathered}
$$

The system noise covariance matrix and the measurement noise covariance matrix where set by trial and error.

$$
\begin{aligned}
& Q(k)=\left[\begin{array}{ccc}
10^{-2} & 0 & 0 \\
0 & 10^{-2} & 0 \\
0 & 0 & 10^{-2}
\end{array}\right] \\
& R(k)=\left[\begin{array}{ccc}
1 \times 10^{-4} & 0 & 0 \\
0 & 1 & 0 \\
0 & 0 & 1 \times 10^{-8}
\end{array}\right] .
\end{aligned}
$$

Measured signals have different accuracy, which are represented on the measurement covariance matrix. As above mentioned, the angular velocity was obtained by derivation of the angular position. On account of the derivation procedure, the signal of the angular velocity became noisy, hence it has got a big value in the matrix.

Under normal conditions the estimated state vector $\hat{X}$ from the EKF converges to the system state vector $X(k)$ such as all elements of the residual vector $e(k)$ will be relatively small.

The residual vector can be described

$$
\mathrm{e}(k)=Z(k)-H \hat{X}^{-}(k)
$$

It causes a fault, hence one or more system parameters change, and the estimated state vector diverges from the actual state trajectory. The residual vector will increase.

\section{Experimental Results}

In the following the discrete state space model of the electromechanical actuator valve, was used by the Kalman filter to estimate the three states, the motor current, valve angular velocity and angular position. We will show how the simulation results collaborate with the experimental data. Both the throttle valve model and the extended Kalman filter algorithm are computed in MATLAB ${ }^{\circledR}$ SIMULINK ${ }^{\circledR}$. The experimental measurements were made using National Instruments CompactRIO ${ }^{\circledR} 9014$ system. The 
actuator was excited through PWM-Signal with square wave of $1 \mathrm{kHz}$ and an Amplitude of $12 \mathrm{~V}$.

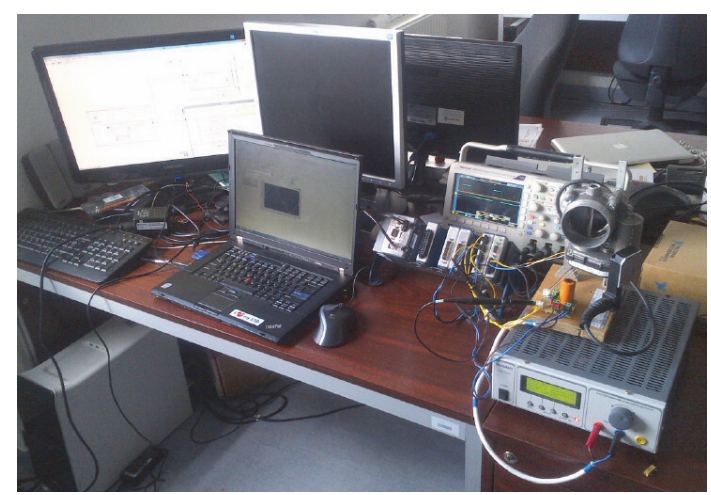

Figure 4. Experimental setup

Input signal for the parameter estimation was a sine wave with frequence of $5 \mathrm{~Hz}$ and amplitude of $12 \mathrm{~V}$. The measured data (motor current, valve angular position) where recorded with sampling time of $1 \mathrm{~ms}$. The block diagram of the experimental setup is shown on the Fig. 5. Since the throttle valve was not equipped with any velocity sensor, the angular velocity of the throttle plate has been calculated by differentiating of the measured values of the angular position. Henceforth the angular velocity is treated as that where measured.

The measured data was used by the EKF to estimate the three states, the approach works iterative. Finally the estimated results were compared with data obtained from simulation of the Actuator model.

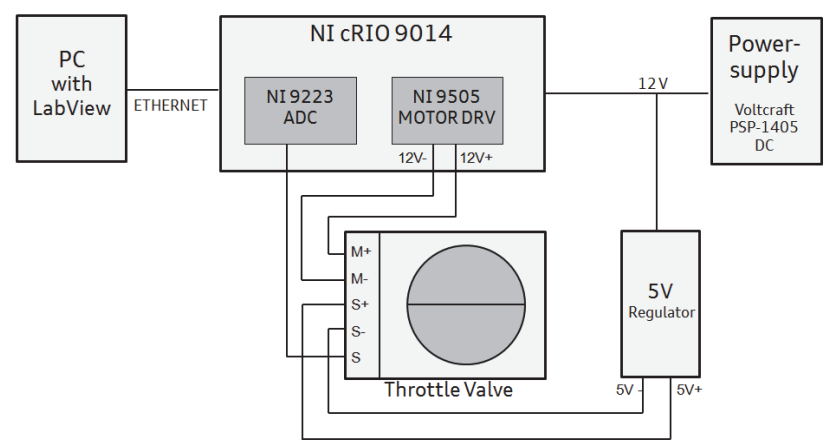

Figure 5. Block diagram of the experimental setup

The initial state vector and the initializing matrices for the EKF were set as mentioned in chapter 3.3.

The graphs in the Figures 6, 7, 8 show the estimated state curves with simulated ones. It can be seen that the results collaborate well with the results of the simulated data. 

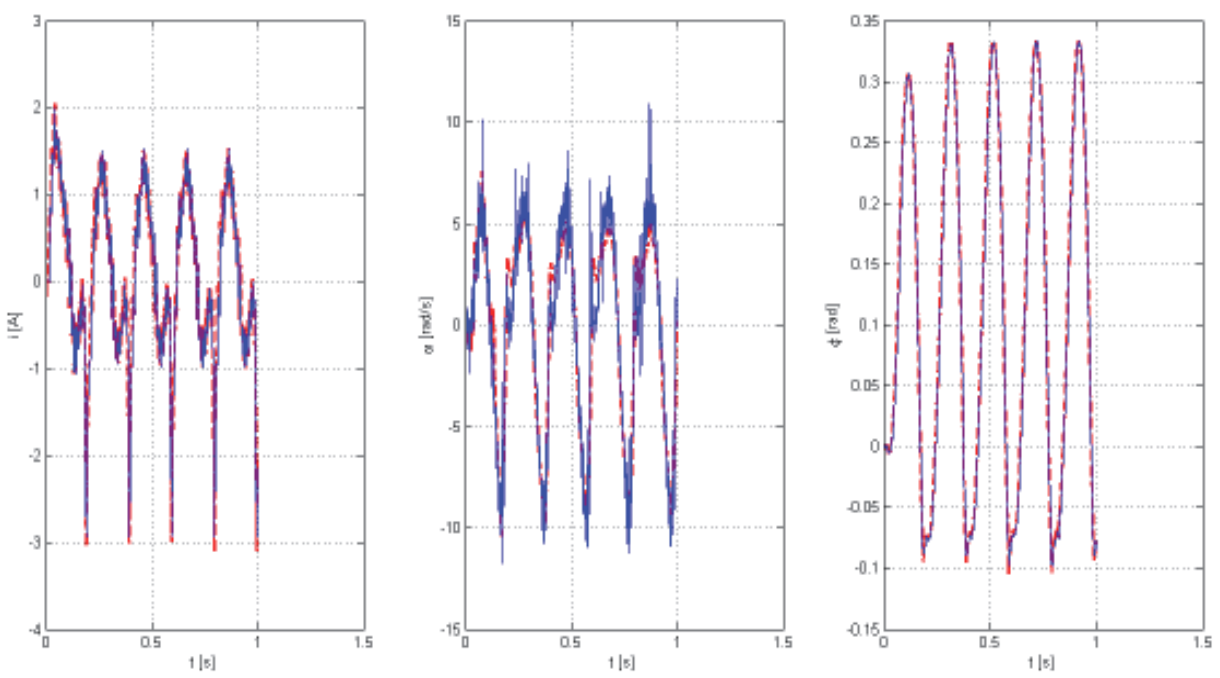

Figure 6. Experimental results measured vs. estimated states of the throttle valve blue line: measured state, red dashed line : estimated state,

left: motor current, middle : angular velocity, right: angular position of the valve
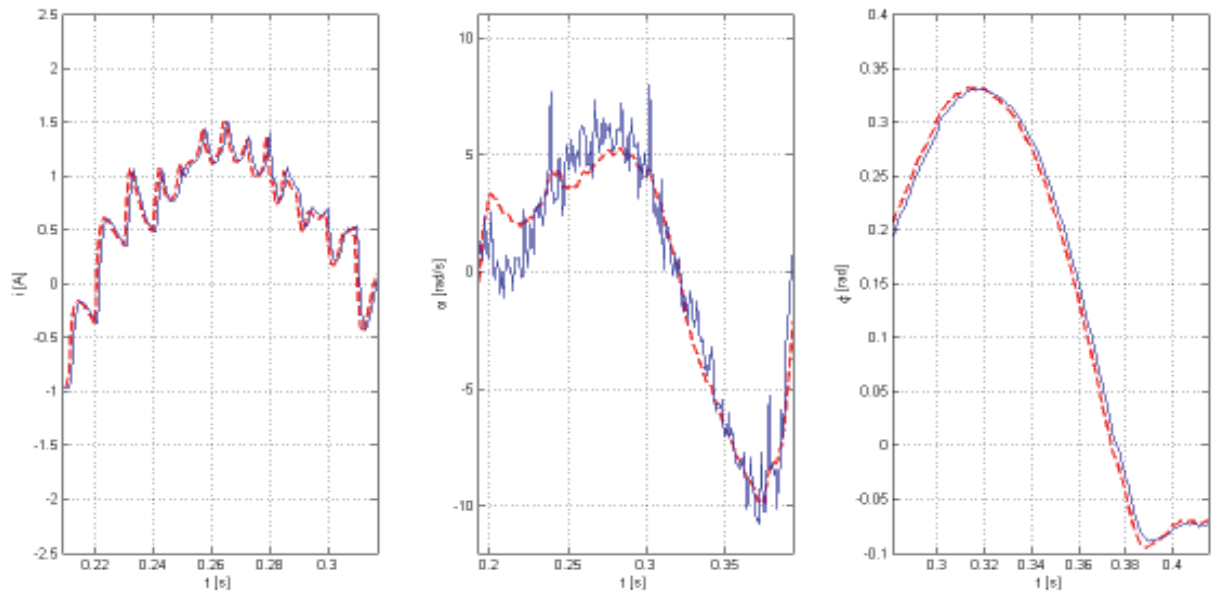

Figure 7. Comparison measured and estimated states of the throttle valve blue line: measured state, red dashed line : estimated state,

left: motor current, middle : angular velocity, right: angular position of the valve

As we can see it in the Figures the estimated values of the states, motor current and valve angular position matches the measured states very closely as it is proved by the curve of the residual too. The estimated angular velocity matches not so good with the mesaured data curves. The reason for this is, that the angular velocity was not measured, just obtained by derivation of the angular position. The measured data of the velocity was not filtered. The initial matrices used for initializing the EKF were changed 
before and it was discorvered that the estimation of the angular velocity was significantly affected. A best fit between the estimated and simulated curves was obtained by taking initial matrices as presented in chapter 3.3. Fig. 8 shows the residue signal of the motor current - and angular position. It can be seen, that the curve of motor current is different from zero. That can be caused by uncertainties of the model parameters. In spite of them, the EKF estimates both motor current and valve angular position successful.
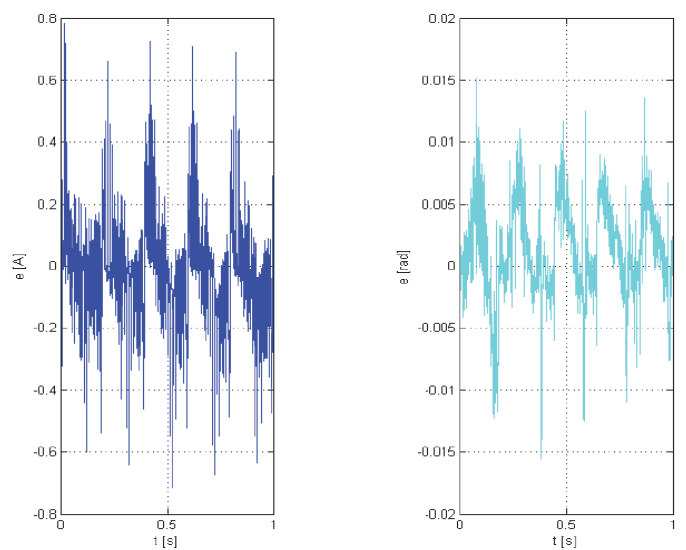

Figure 8. Residue computed during EKF iterations

blue line: motor current, cian dashed line : angular position of the valve

\section{Conclusion}

The state space estimation for an electromechanical actuator valve using extended Kalman filter (EKF) is discussed in this paper. Our actuator model captures the Tustin's friction model which includes nonlinearities. It is necessary because the EKF requires an accurate model of the plant. The first step before applying the EKF was converting the state equations of the actuator model into a time-discrete. Than the EKF was constructed and used to estimate three states of the system, namely the motor current, valve angular velocity - and position. After this the described actuator model and the EKF algorithm were implemented in MATLAB ${ }^{\circledR}$. The measurements for the input data for the EKF were performed in National Instruments CompactRIO® 9014 system.

The curve of the residual proves, that the estimated values of the states, motor current and valve angular position matches the measured states very closely.

With our examination we have proved, that the proposed iterative EKF approach is able to estimate the states of the electromechanical actuator valve with strong nonlinearities successfully. 


\section{References}

[1] Nakano K, Sawut U, Higuci K, Okajima Y: Modelling an Observer-based SlidingMode Control of Electronic Throttle Systems. ECTI Transactions on Electrical Eng., Electronics, and Communications, Vol. 4, No. 1, 2006.

[2] Reichhartinger M, Horn M: Robust Position Control of an Electromechanical Actuator for Automotive Applications. World Academy of Science, Engineering and Technology, Vol. 5, 2011.

[3] Reinelt W, Lundquist C: Observer Based Sensor Monitoring in Active Front Steering System using Explicit Sensor Failure Modelling. Proceedings of the $16^{\text {th }}$ IFAC World Congress, Vol. 16, No. 1, 2005.

[4] Padmakumar S, Vivek A, Kallol R: A Comparative Study into Observer based Fault Detection and Diagnosis in DC Motors: Part-I. World Academy of Science, Engineering and Technology, Vol. 3, No. 3, 2009.

[5] Vašak M, Petrović I, Perić N: State Estimation of an Electronic Throttle Body. Industrial Technology IEEE International Conference, Vol.1, pp. 472-477, 2003.

[6] Wright G: Parameter Estimation of a Hydraulic Proportional Valve Using Extended Kalman Filtering. M.Sc. Thesis, University of Saskatchewan, Canada, 2001.

[7] Yuvin AC: Fault Detection In the Electrohydraulic Actuator Using Extended Kalman Filter. Doctoral Thesis, University of Saskatchewan, Canada, 2004.

[8] Grepl R, Lee B: Modelling, Identification and Control of Electronics Throttle Body Using Dspace Tools. Technical Computing Prague, 2008.

[9] Loh RNK, Thanom W, Pyko JS, Lee A: Electronic Throttle Control System: Modeling, Identification and Model-Based Control Designs. Engineering, Vol. 5, No. 7, pp. 587-600, 2013.

DOI: $10.4236 /$ eng. 2013.57071

[10] Horváth Zs, Molnárka Gy: Design Luenberger Observer for an Electromechanical Actuator. Acta Technica Jaurinensis, Vol. 7, No. 4, 2014.

DOI: 10.14513/actatechjaur.v7.n4.313

[11] Nor Hazadura H, Sazali Y, Hariharan M, Norhizam H: Nonlinear observers for attitude estimation in gyroless spacecraft via Extended Kalman filter algorithm. International Journal of Scientific and Research Publications, Vol. 4, 2014.

[12] Slotine J-JE, Li W: Applied Nonlinear Control. Prentice Hall, Englewood Cliffs, New Jersey, pp. 229-234, 1991. 